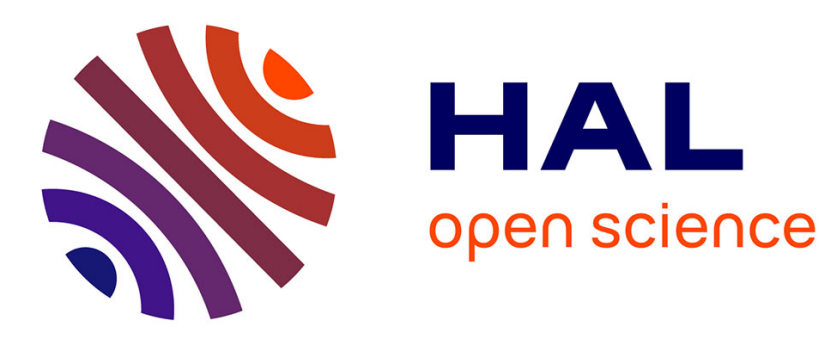

\title{
Surface Phonon-Polariton Heat Capacity of Polar Nanofilms
}

\author{
Jose Ordonez-Miranda, Sebastian Volz, Masahiro Nomura
}

\section{To cite this version:}

Jose Ordonez-Miranda, Sebastian Volz, Masahiro Nomura. Surface Phonon-Polariton Heat Capacity of Polar Nanofilms. Physical Review Applied, 2021, 15, 10.1103/physrevapplied.15.054068 . hal03420113

\section{HAL Id: hal-03420113 \\ https://hal.science/hal-03420113}

Submitted on 9 Nov 2021

HAL is a multi-disciplinary open access archive for the deposit and dissemination of scientific research documents, whether they are published or not. The documents may come from teaching and research institutions in France or abroad, or from public or private research centers.
L'archive ouverte pluridisciplinaire HAL, est destinée au dépôt et à la diffusion de documents scientifiques de niveau recherche, publiés ou non, émanant des établissements d'enseignement et de recherche français ou étrangers, des laboratoires publics ou privés. 
See discussions, stats, and author profiles for this publication at: https://www.researchgate.net/publication/351953884

\section{Surface Phonon-Polariton Heat Capacity of Polar Nanofilms}

Article in Physical Review Applied · May 2021

DOI: 10.1103/PhysRevApplied.15.054068

CITATION

3 authors:

Jose Ordonez-Miranda

CNRS

143 PUBLICATIONS 1,680 CITATIONS

SEE PROFILE

17in. Masahiro Nomura

[1] The University of Tokyo

195 PUBLICATIONS 3,046 CITATIONS

SEE PROFILE

Some of the authors of this publication are also working on these related projects:

Project Thermal Phononics View project

Project Thermoelasticity View project
READS

163

Sebastian Volz

French National Centre for Scientific Research

364 PUBLICATIONS 7,378 CITATIONS

SEE PROFILE 


\title{
Surface Phonon-Polariton Heat Capacity of Polar Nanofilms
}

\author{
Jose Ordonez-Miranda $\odot,{ }^{1,2, *}$ Sebastian Volz $\odot,{ }^{1,2, \dagger}$ and Masahiro Nomura $\odot^{2, \ddagger}$ \\ ${ }^{1}$ LIMMS, CNRS-IIS UMI 2820, The University of Tokyo, Tokyo 153-8505, Japan \\ ${ }^{2}$ Institute of Industrial Science, The University of Tokyo, Tokyo 153-8505, Japan
}

(Received 17 March 2021; revised 25 April 2021; accepted 14 May 2021; published 28 May 2021)

\begin{abstract}
The surface phonon-polariton heat capacity of polar nanofilms is analytically determined and analyzed as a function of their thickness and temperature. It is shown that the polariton heat capacity increases with the square of the nanofilm thickness, such that for thin enough nanofilms at sufficient low temperature $T$, its value becomes independent of the material properties and is given by $C=1.15 \varepsilon_{0} k_{B}\left(k_{B} T / \hbar c\right)^{2}$, where $\varepsilon_{0}$ and $c$ are the respective relative permittivity of the surrounding medium and light speed in vaccum, while $k_{B}$ and $2 \pi \hbar$ are the Boltzmann and Planck constants, respectively. The photonlike nature of surface phonon polaritons is found to be responsible for their main contribution to the material heat capacity. As a result of the polariton speed close to $c$ and hence much higher than that of phonons, the polariton heat capacity of $\mathrm{SiO}_{2}, \mathrm{SiC}$, and $\mathrm{SiN}$ is found to be several orders of magnitude smaller than its corresponding phonon counterpart. Surface phonon polaritons are thus not expected to increase the expansion coefficient of polar nanofilms, which favors their utilization as effective heat dissipators.
\end{abstract}

DOI: 10.1103/PhysRevApplied.15.054068

\section{INTRODUCTION}

Surface phonon polaritons (SPHPs) are surface electromagnetic waves generated by phonon-photon coupling at the interface of polar materials, such as $\mathrm{SiO}_{2}, \mathrm{SiC}$, and $\mathrm{SiN}$ [1-13]. This coupling blurs the boundary between light and heat, occurs at midinfrared frequencies mainly, and triggers the propagation of SPHPs over distances (approximately $1 \mathrm{~cm}$ ) orders of magnitude longer than the typical mean free paths of phonons and electrons [14-16]. These long-range surface waves have thus emerged as powerful heat carriers able to enhance the in-plane thermal conductivity of polar materials with high surface-to-volume ratios, as is the case of nanofilms and nanostructures with usually low thermal performances $[17,18]$.

The SPHP contribution to the thermal conductivity of polar nanofilms was modeled and quantified by Chen et al. [1], who reported a thermal conductivity of $4 \mathrm{Wm}^{-1} \mathrm{~K}^{-1}$ for a 40 -nm-thick $\mathrm{SiO}_{2}$ film suspended in vacuum at 500 $\mathrm{K}$. This prediction of the kinetic theory is more than twice the intrinsic phonon thermal conductivity of $\mathrm{SiO}_{2}$, was confirmed by means of the fluctuation-dissipation approach [4], and extended for films deposited over a substrate [2], other polar materials [19], layered systems [20-22], and arrangement of particles [23-25]. On the experimental side, on the other hand, Tranchant et al. [26]

\footnotetext{
*ordonez@iis.u-tokyo.ac.jp

†volz@iis.u-tokyo.ac.jp

‡nomura@iis.u-tokyo.ac.jp
}

measured the in-plane thermal conductivity of suspended $\mathrm{SiO}_{2}$ nanofilms and showed up its clear increase as the film thickness reduces through values smaller than $50 \mathrm{~nm}$. More recently, by measuring the temperature evolution of the in-plane thermal conductivity of SiN nanofilms, $\mathrm{Wu}$ et al. [5] found that its values doubles up as the temperature raises from 300 to $800 \mathrm{~K}$. This thermal conductivity enhancement for thinner and hotter polar nanofilms represents the fingerprints of the SPHP heat transport $[1,2]$ and hence its experimental observation $[5,26]$ provides decisive evidence for the potential application of SPHPs as long-distance heat dissipators.

Heat conduction under dynamical conditions is not only driven by the thermal conductivity but also by the thermal diffusivity and heat capacity, which is proportional to the expansion coefficient that affects the structural stability of heat dissipation [27]. Experimental measurements showed that the thermal diffusivity of polar nanofilms increases as their thicknesses decrease [6,26]. This thickness dependence is similar to the one of the thermal conductivity of polar nanofilms $[5,26]$, which suggests that their heat capacity (which equals thermal conductivity divided by thermal diffusivity) could be independent or weakly dependent on the film thickness. While the phonon and electron contributions to the heat capacity are well detailed in textbooks [27,28], the one of SPHPs has not been studied yet.

In this work, the SPHP heat capacity of polar nanofilms is analytically modeled, quantified, and analyzed as a function of their thickness and temperature. It is shown that the SPHP heat capacity is independent of the material 
properties and proportional to the square of temperature, for thin enough nanofilms at sufficiently low temperatures.

\section{THEORETICAL MODEL}

Let us consider a polar film of thickness $d$, length $a$, and width $b$ supporting the propagation of SPHPs, as shown in Fig. 1. These SPHPs can be generated by thermal or optical means [29-33] and their propagation is strongly determined by the permittivity $\varepsilon$ and thickness $d$ of the film $[1,2]$. Given that the generation of SPHPs is driven by the phonon-photon coupling that depends on the external excitation, their propagation and energy transport are determined by the type of source that induces them. While the optical excitation consists in illuminating the material surface with a monochromatic light beam of a given frequency and hence allows exciting a single SPHP mode at a time $[9,29]$, the thermal one is based on heating the material and simultaneously generating SPHPs in a wide frequency spectrum [4,34], which is required to enhance their heat transport [1,2]. In the present work, we assume that the SPHPs are thermally excited to determine the SPHP heat capacity from a broad frequency range supporting their propagation, as shown below.

Taking into account that the SPHPs can be treated like a gas of bosonic particles with energy $\hbar \omega$, as is the case of photons and phonons, their contribution to the internal thermal energy per unit area of the film at temperature $T$ is given by [27]

$$
U=\int \hbar \omega f(T, \omega) D(\omega) d \omega
$$

where $\hbar$ is Planck's constant divided by $2 \pi, f=$ $\left[\exp \left(\hbar \omega / k_{B} T\right)-1\right]^{-1}$ is the Bose-Einstein distribution function, and $D(\omega)$ is the SPHP density of states per unit frequency $\omega$. Since SPHPs mainly propagate along the interface of the film and they span over its surface, their density of states is two dimensional (2D) and is given by $[1,34] D(\omega)=\beta_{R} /(2 \pi V)$, with $\beta_{R}=\operatorname{Re}(\beta)$ and $V=$ $\partial \omega / \partial \beta_{R}$ being the real part of the in-plane wave vector $\beta$

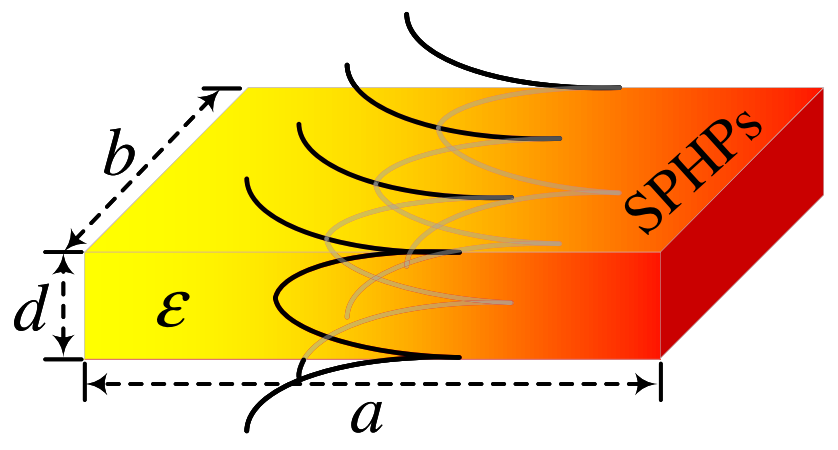

FIG. 1. Scheme of a polar nanofilm supporting the propagation of surface phonon polaritons. and group velocity of SPHPs, respectively. The SPHP contribution to the heat capacity per unit surface of the film can then be determined by $C=\partial U / \partial T[27,28]$, which in combination with Eq. (1) yields

$$
C=\frac{1}{2 \pi} \int \frac{\hbar \omega \beta_{R}}{V} \frac{\partial f}{\partial T} d \omega
$$

In writing Eq. (2), we assume that the wave vector $\beta_{R}=$ $\beta_{R}(\omega, \varepsilon, d)$ is independent of temperature. This assumption is valid within a wide interval of temperatures below and above room temperature, as is the case of the permittivity $\varepsilon$ of $\mathrm{SiO}_{2}, \mathrm{SiC}$, and $\mathrm{SiN}[5,35]$. Equation (2) thus establishes that $C$ depends on the material properties through the ratio $\beta_{R} / V$, which can be calculated from the dispersion relation of SPHPs propagating along the polar film, as shown in Fig. 1. When the film thickness $d$ is small enough $\left(\lambda=k_{0} d / 2 \ll 1\right)$, which is the case of interest for enhancing their thermal energy transport $[1,2,5,26]$, one can show that the real and imaginary parts of the SPHP wave vector $\beta=\beta_{R}+i \beta_{I}$ are well approximated by $[2,5]$

$$
\begin{aligned}
& \beta_{R}=k_{0} \sqrt{\varepsilon_{0}}\left(1+A \lambda^{2}\right), \\
& \beta_{I}=k_{0} \sqrt{\varepsilon_{0}} \frac{\varepsilon_{0}^{2} \operatorname{Im}[\varepsilon(\omega)]}{|\varepsilon(\omega)|^{2}} J \lambda^{2},
\end{aligned}
$$

where $\varepsilon_{0}$ is the relative permittivity of the medium surrounding the film, $k_{0}=\omega / c, c$ is the speed of light in vacuum, and

$A=\frac{\varepsilon_{0}}{2}\left(1-\frac{2 \varepsilon_{0} \operatorname{Re}[\varepsilon(\omega)]}{|\varepsilon(\omega)|^{2}}+\frac{\varepsilon_{0}^{2}\left\{\operatorname{Re}[\varepsilon(\omega)]^{2}-\operatorname{Im}[\varepsilon(\omega)]^{2}\right\}}{|\varepsilon(\omega)|^{4}}\right)$,

$J=1-\frac{\varepsilon_{0} \operatorname{Re}[\varepsilon(\omega)]}{|\varepsilon(\omega)|^{2}}$.

Taking into account that the SPHP propagation length is given by $[2,16] \Lambda=\left(2 \beta_{I}\right)^{-1}$, the key function $J(\omega)$ establishes that the SPHPs propagate only for frequencies that satisfy the condition $J>0$. The positive values of $J(\omega)$ also determine the confinement of SPHPs to the film interface [5], and therefore they can be used to quantify the frequency spectrum $(J>0)$ supporting the existence and propagation of SPHPs in different polar nanofilms. Based on Eq. (3a), one can show that $\beta_{R} / V=$ $\left(k_{0} \varepsilon_{0} / c\right)\left[1+(4 A+\omega \partial A / \partial \omega) \lambda^{2}\right]$, which allows rewriting Eq. (2) as follows:

$$
C=C_{0}+C_{2} d^{2}
$$

where the coefficients $C_{0}$ and $C_{2}$ are defined by

$$
C_{0}=\frac{\varepsilon_{0}}{2 \pi c^{2}} \int \hbar \omega^{2} \frac{\partial f}{\partial T} d \omega,
$$




$$
C_{2}=\frac{\varepsilon_{0}}{8 \pi c^{4}} \int \hbar \omega^{4}\left(4 A+\omega \frac{\partial A}{\partial \omega}\right) \frac{\partial f}{\partial T} d \omega .
$$

Equation (5) thus establishes that the SPHP heat capacity generally increases with the square of the film thickness, within the thin-film limit $\left(k_{0} d / 2 \ll 1\right)$. In practice, this condition is typically fulfilled for $d<300 \mathrm{~nm}[2,3]$, which indicates that $C$ can become independent of the properties of films with thicknesses of tens of nanometers, as is established by Eqs. (5) and (6a) $\left(C \rightarrow C_{0}\right)$. Note that both parameters $C_{0}$ and $C_{2}$, and hence $C$, increase with the permittivity $\varepsilon_{0}$ of the surrounding medium. This fact indicates that $C$ exhibits an opposite dependence on $d$ and $\varepsilon_{0}$ than its SPHP thermal conductivity, which is lower for greater values of $d$ and $\varepsilon_{0}$ [2]. According to its definition $(C=\partial U / \partial T)$, the SPHP heat capacity per unit area $C\left(\mathrm{~J} \mathrm{~m}^{-2} \mathrm{~K}^{-1}\right)$ is the amount of heat required to raise the temperature of the film material with an arbitrary thickness by $1 \mathrm{~K}$, and therefore its increase with $d$ is reasonable, as established by Eq. (5). By contrast, the corresponding SPHP specific heat capacity $c=a b C /$ mass $=$ $a b C /(\rho a b d)=C /(\rho d)=\left(C_{0} d+C_{2} d\right) \rho$, with $\rho$ being the film density, takes higher values for thinner films, as is the case of the SPHP thermal conductivity. The surface nature of SPHPs can thus be seen in both its thermal conductivity and specific heat capacity (intensity property), whose values are higher for polar materials with higher surface-to-volume ratios.

In order to show up the temperature behavior of the fundamental component $C_{0}$, Eq. (6a) is rewritten as follows:

$$
C_{0}=\frac{\varepsilon_{0} k_{B}}{2 \pi}\left(\frac{k_{B} T}{\hbar c}\right)^{2} \int_{0}^{\theta / T} \frac{x^{3} e^{x}}{\left(e^{x}-1\right)^{2}} d x,
$$

where $x=\hbar \omega / k_{B} T$ and $\theta=\hbar \omega_{\max } / k_{B}$ is a characteristic temperature determined by the maximum frequency $\omega_{\max }$ supporting the propagation of SPHPs. The integrand in Eq. (7) establishes that the heat capacity spectrum of a polar nanofilm at temperature $T$ peaks at the frequency $\omega_{p}$ given by $\hbar \omega_{p} / k_{B} T=2.5757$, that is to say, $\omega_{p} / 2 \pi=$ $0.05367 T\left(\mathrm{THz} \mathrm{K}^{-1}\right)$, which takes the value of $16.1 \mathrm{THz}$ at room temperature. Note that Eq. (7) is analogous to the Debye model for the phonon heat capacity, with $\theta$ being the Debye temperature for SPHPs. At high temperature $(T \gg \theta)$, the integral in Eq. (7) reduces to $0.5(\theta / T)^{2}$ and $C_{0}$ takes its maximum value

$$
C_{0, \max }=\frac{\varepsilon_{0} k_{B}}{4 \pi}\left(\frac{k_{B} \theta}{\hbar c}\right)^{2}=\frac{\varepsilon_{0} k_{B}}{4 \pi}\left(\frac{\omega_{\max }}{c}\right)^{2} .
$$

The dependence of $C_{0, \max }$ on $\omega_{\max }^{2}$ is the result of the 2D density of states of SPHPs, which is analog to the dependence on $\omega_{\max }^{3}$ of the high-temperature heat capacity of phonons, due to their three-dimensional (3D) density of

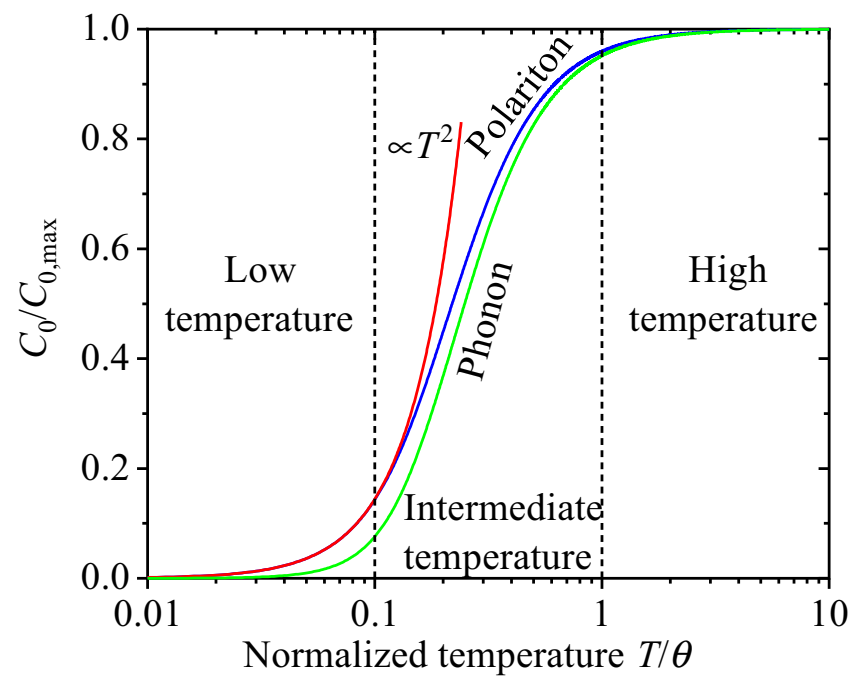

FIG. 2. Fundamental component of the SPHP heat capacity of a polar nanofilm as a function of its temperature. The red line represents the low-temperature limit predicted by Eq. (9) and the green one stands for the prediction of the Debye model for phonons.

states, as predicted by the Debye model [27,28]. For low temperatures $(T \ll \theta)$, on the other hand, the integral in Eq. (7) has the value $6 z(3)$ of 6 times the Riemann $\zeta$ function $z(3)$ and $C_{0}$ increases with the square of temperature, as follows:

$$
C_{0}=\frac{3 z(3)}{\pi} \varepsilon_{0} k_{B}\left(\frac{k_{B} T}{\hbar c}\right)^{2}
$$

Equations (7)-(9) thus indicate that the SPHP heat capacity of a polar nanofilm is strongly determined by the relative values of its temperature with respect to the characteristic one $\theta$, as shown in Fig. 2. Note that the normalized SPHP heat capacity follows pretty much the same behavior as its phonon counterpart [27,28], through the three regimes determined by the ratio $T / \theta$. The $T^{2}$ regime in which $C_{0}$ is independent of the material properties, shows up for $T / \theta \leq 0.1$, while $C_{0}$ reaches its maximum value for $T / \theta>1$.

\section{RESULTS AND DISCUSSION}

According to Eqs. (3b) and (4b), the range of frequencies supporting the propagation of SPHPs is determined by the condition $(J>0)$

$$
\operatorname{Re}[\varepsilon(\omega)]<\frac{|\varepsilon(\omega)|^{2}}{\varepsilon_{0}},
$$

which can be satisfied not only by negative values of $\operatorname{Re}(\varepsilon)$, but also by positive ones. The key condition in Eq. (10) thus indicates that the SPHP propagation along the 
interface of polar nanofilms is totally determined by its permittivity $(\varepsilon)$ and that $\left(\varepsilon_{0}\right)$ of its surrounding medium, while being independent of its thickness. Note that the range of allowed frequencies reduces to those within the negative peak of $\operatorname{Re}[\varepsilon(\omega)]<0$ (Reststrahlen band) for $\varepsilon_{0} \rightarrow \infty$ only. For any other case of practical interest, the range of frequencies supporting the propagation of SPHPs is usually much broader than the Reststrahlen band, as was reported experimentally $[14,15]$ and is predicted by the function $J>0$. This frequency range is independent of the film thickness [Eq. (10)], provided that the thin-film limit $(\lambda \ll 1)$ is valid, which ensures a strong coupling of the SPHPs propagating along the upper and lower interfaces of the film. In fact, the SPHP modes propagating along lossy nanofilms, as is the case of our present work, are called Zenneck modes, which exist and propagate with frequencies inside and outside the Reststrahlen band, as detailed in the literature $[3,29]$ and are shown in Fig. 4. Furthermore, in absence of energy absorption $[\operatorname{Im}(\varepsilon)=0]$, Eq. (10) reduces to $\operatorname{Re}(\varepsilon)=\varepsilon>\varepsilon_{0}$, which is totally different than the well-known condition $\varepsilon<-\varepsilon_{0}$ determining the propagation of SPHPs along a single interface of a bulk lossless material.

As the function $J$ changes of sign at $\operatorname{Re}[\varepsilon(\omega)]=$ $|\varepsilon(\omega)|^{2} / \varepsilon_{0}$, the cutoff frequency $\omega=\omega_{\max }$ is given by the solution of this latter relation, which can be rewritten as the circle equation $\left\{\operatorname{Re}[\varepsilon(\omega)]-\varepsilon_{0} / 2\right\}^{2}+\{\operatorname{Im}[\varepsilon(\omega)]\}^{2}=$ $\left(\varepsilon_{0} / 2\right)^{2}$. This circle of radius $\varepsilon_{0} / 2$ represents the transition between the range of frequencies that allow (outside the circle) or forbid (inside the circle) the propagation of SPHPs. Therefore, if there is more than one value for $\omega_{\max }$, the integrals in Eqs. (6a) and (6b) have to be integrated skipping the frequency band gaps in which SPHPs do not propagate $(J<0)$.

The frequency dependence of the real $\varepsilon_{R}(\omega)$ and imaginary $\varepsilon_{I}(\omega)$ parts of the relative permittivity of $\mathrm{SiO}_{2}, \mathrm{SiC}$, and $\mathrm{SiN}$ are shown in Figs. 3(a)-3(c), respectively [2,3,5]. Note that $\mathrm{SiO}_{2}$ has two Reststrahlen bands $\left[\varepsilon_{R}(\omega)<0\right]$ determined by the frequency intervals $(13.8 ; 15.1) \mathrm{THz}$ and $(32.1 ; 37.3)$ THz. On the other hand, SiC and $\mathrm{SiN}$ have a single Reststrahlen band each, within the frequency ranges $(23.7 ; 28.9) \mathrm{THz}$ and $(26.6 ; 31.8) \mathrm{THz}$, respectively. For the three considered materials, these frequency bands contain the resonance peaks of $\varepsilon_{R}(\omega)$ and occur in the vicinity of the maxima of $\varepsilon_{I}(\omega)$. This fact indicates that the materials absorb the highest amount of energy from the electromagnetic field at frequencies near the ones defining their corresponding Reststrahlen bands. In these bands, the function $J$ (Fig. 4) is positive and therefore they support the existence and propagation of SPHPs. Note that both $\mathrm{SiO}_{2}$ and $\mathrm{SiC}$ exhibit frequency band gaps (colored areas) just above their corresponding Reststrahlen bands. By contrast, these band gaps are absent for the SiN film, which hence is capable of supporting the SPHP propagation for all considered frequencies.
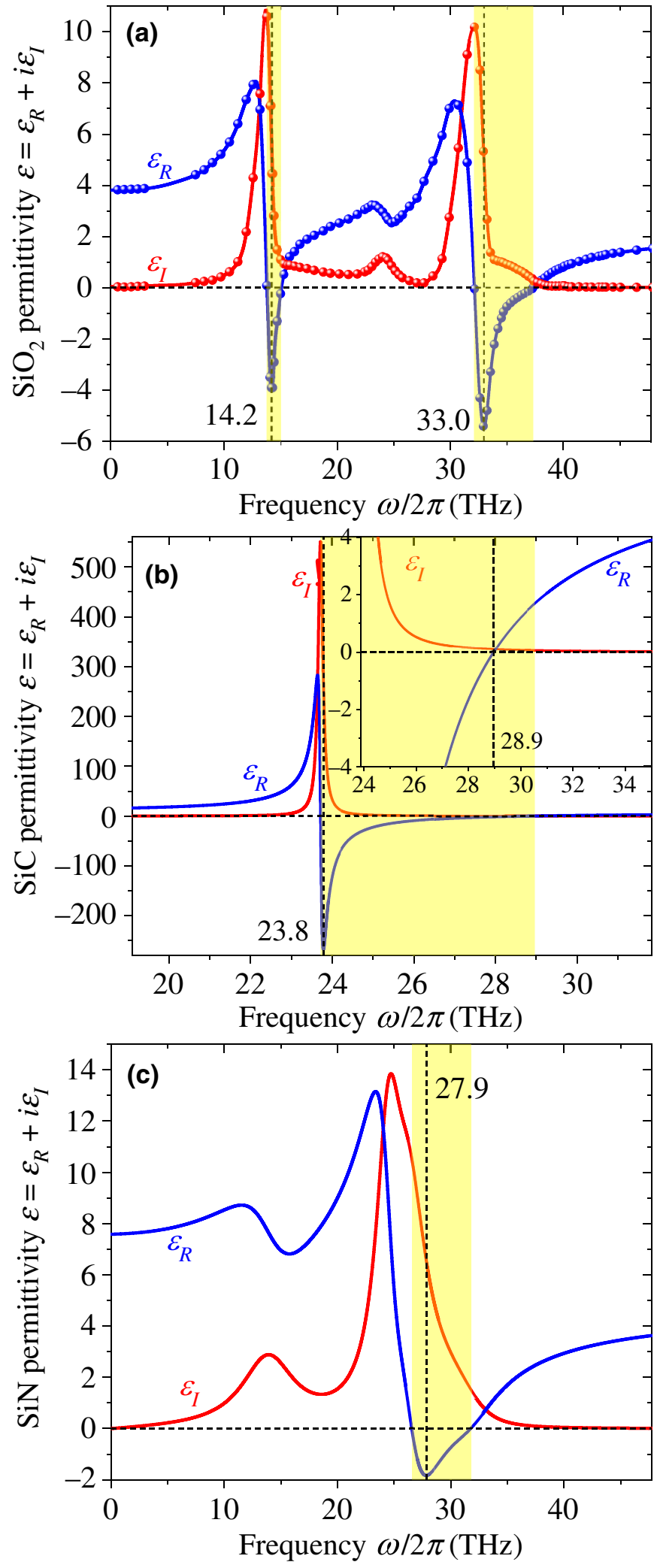

FIG. 3. Real and imaginary parts of the relative permittivity $\varepsilon(\omega)=\varepsilon_{R}(\omega)+i \varepsilon_{I}(\omega)$ of (a) $\mathrm{SiO}_{2}$, (b) $\mathrm{SiC}$, and (c) $\mathrm{SiN}$ as a function of frequency $[2,3,5]$. The yellow zones stand for the Reststrahlen bands $\left[\varepsilon_{R}(\omega)<0\right]$ of each material. 


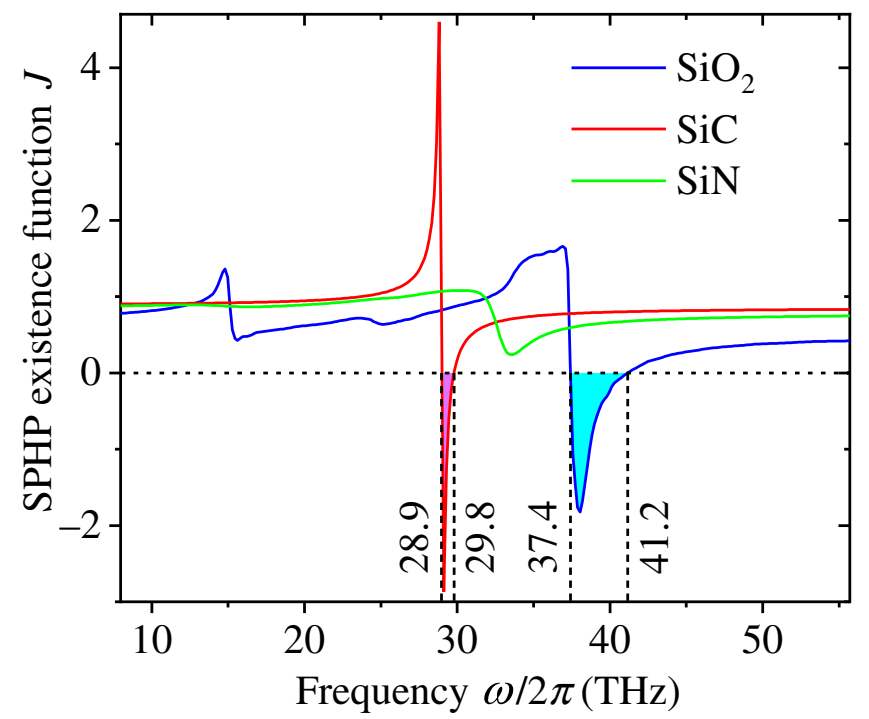

FIG. 4. Frequency spectrum of the SPHP existence function of three common polar materials surrounded by vacuum $\left(\varepsilon_{0}=1\right)$. The colored areas represent the band gaps for the existence of SPHPs and the calculations are done with the complex permittivities of $\mathrm{SiO}_{2}, \mathrm{SiC}$, and $\mathrm{SiN}$ reported in the literature $[2,3,5]$.

The frequency dependence of the dispersion relation (real part $\beta_{R}$ of the in-plane wave vector) and propagation length $\Lambda$ of SPHPs propagating along the interfaces of 100-nm-thick films of $\mathrm{SiO}_{2}, \mathrm{SiC}$, and $\mathrm{SiN}$ suspended in air, are shown in Figs. 5(a) and 5(b), respectively. For the three materials, $\beta_{R}$ linearly increases with frequency through values very similar to those of the light line, in particular, for $\omega / 2 \pi<250 \mathrm{THz}$. In this frequency regime, the SPHPs thus show a photonlike behavior characterized by a group velocity close to that of light in vacuum $(V \rightarrow c)$. For higher frequencies, on the other hand, the SPHP velocity decreases as a result of the values of $\beta_{R}$ higher than those of the light line. The fact that $\beta_{R}(\mathrm{SiC})>\beta_{R}(\mathrm{SiN})>$ $\beta_{R}\left(\mathrm{SiO}_{2}\right)$ establishes that SPHPs propagate in $\mathrm{SiC}$ slower than in $\mathrm{SiN}$ and $\mathrm{SiO}_{2}$. However, $\mathrm{SiC}$ supports SPHPs with longer propagation lengths $(\Lambda>1 \mathrm{~mm})$ than these latter two materials, within a representative range of frequencies, as shown in Fig. 5(b). Note that both $\mathrm{SiC}$ and $\mathrm{SiO}_{2}$ exhibit frequency band gaps (colored regions) for which there is no propagation of SPHPs, in agreement with the predictions of the SPHP existence function shown in Fig. 4. For the three materials, the maxima and minima of $\Lambda$ appear at frequencies close to the corresponding ones determining the minima and maxima of the imaginary part of their permittivity [2,3,5], which drives the absorption of energy by the film material. This slight displacement of the peaks and dips of $\Lambda$ and $\operatorname{Im}(\varepsilon)$ is reasonable, given that the SPHP propagation length is not determined only by $\operatorname{Im}(\varepsilon)$, but rather by $\operatorname{Re}(\varepsilon)$, as established by Eq. (3b). The absence of SPHP propagation for the band gaps of $\mathrm{SiN}$ and $\mathrm{SiO}_{2}$
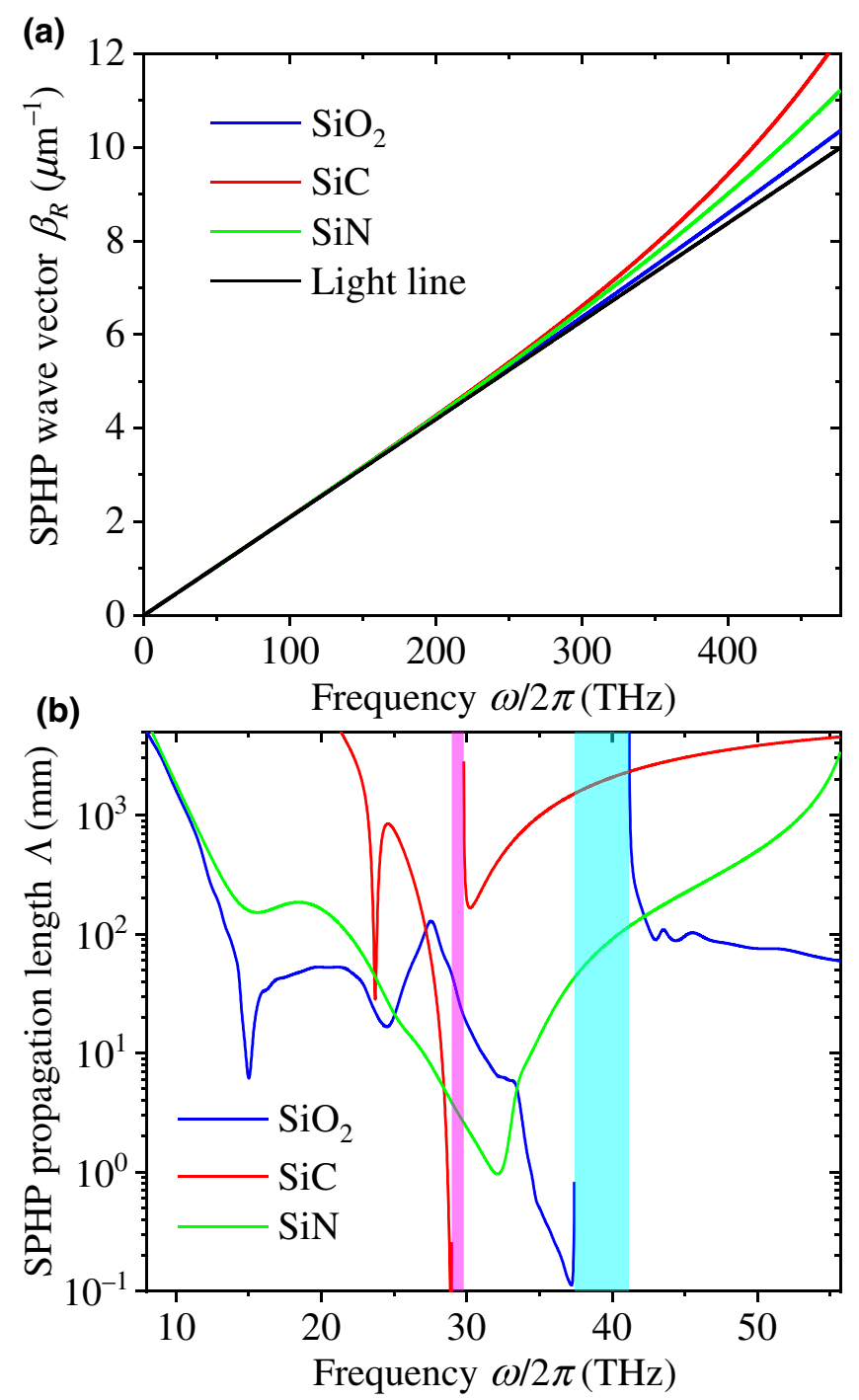

FIG. 5. (a) SPHP dispersion relation and (b) propagation length as functions of frequency, for 100-nm-thick films of $\mathrm{SiO}_{2}$, $\mathrm{SiC}$, and $\mathrm{SiN}$ suspended in air $\left(\varepsilon_{0}=1\right)$. The colored regions represent the range of frequencies for which there is no propagation of SPHPs.

indicates that the SPHP heat capacity of these materials must be determined by excluding their frequency gaps in the integral of Eq. (2).

Figure 6(a) shows the SPHP heat-capacity spectrum established by Eq. (2) and the results shown in Fig. 5 for the films of $\mathrm{SiO}_{2}, \mathrm{SiC}$, and $\mathrm{SiN}$. Note that the spectra for these three materials are pretty much the same at room temperature $(T=300 \mathrm{~K})$ and therefore their SPHP heat capacity $C$ becomes independent of the material properties, as predicted by Eq. (9) and is shown in Figs. 6(b) and 6(c), for thin enough $(d<500 \mathrm{~nm})$ films at low temperature $(T<300 \mathrm{~K})$. By contrast, at high temperature $(T=1000 \mathrm{~K})$, the spectrum for $\mathrm{SiC}$ is higher than those of $\mathrm{SiN}$ and $\mathrm{SiO}_{2}$ due to its relatively high wave vector $\beta_{R}$ 

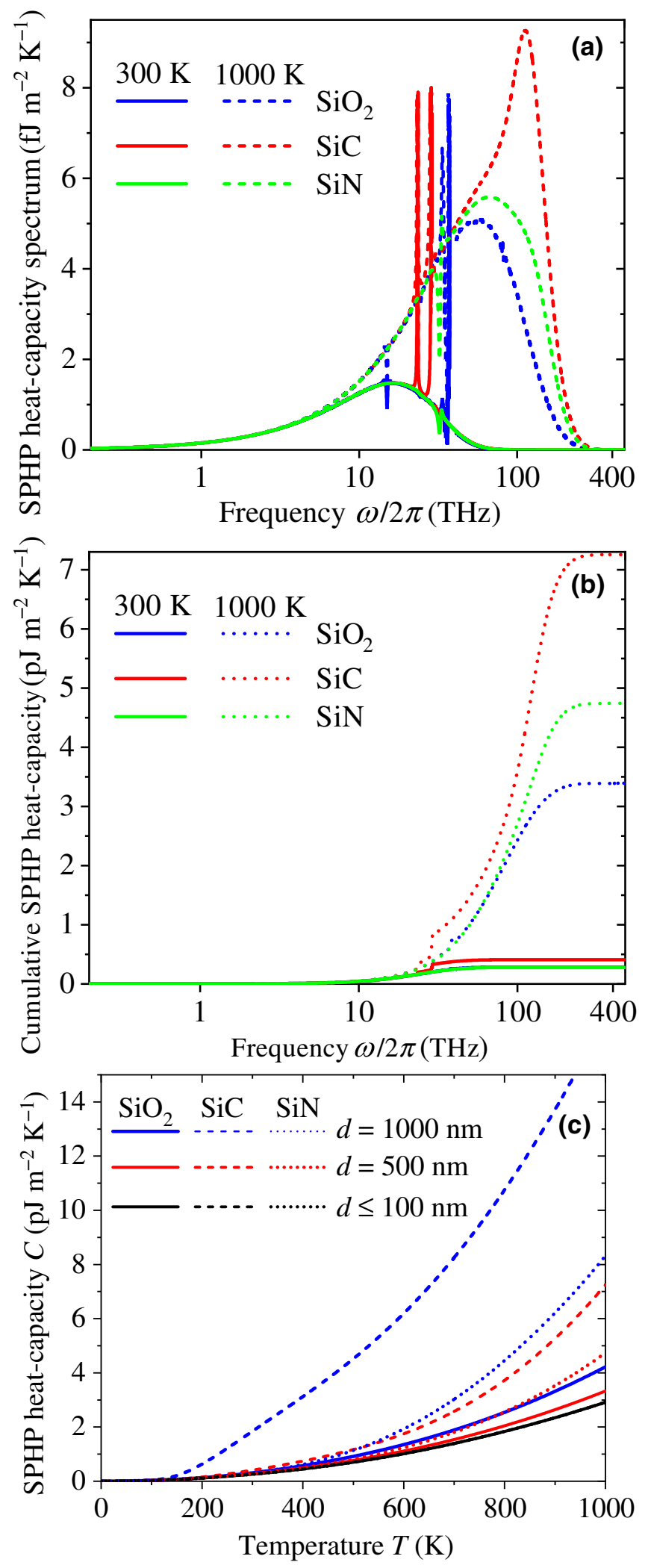

FIG. 6. (a) SPHP heat-capacity spectrum along with its (b) cumulative and (c) integrated values as functions of the temperature of a film suspended in air. Calculations in (a),(b) are done for $d=500 \mathrm{~nm}$, two representative temperatures, and three materials. and slow speed of SPHPs, as shown in Fig. 5(a). According to the cumulative heat capacity in Fig. 6(b), the major contribution to $C$ arises from frequencies $\omega / 2 \pi<200 \mathrm{THz}$ in which the SPHPs propagate with pretty much the same wave vector (and speed) than light, as shown in Fig. 5(a). The photonlike nature of SPHPs is thus responsible of their main contribution to the material heat capacity. In addition, given that $C$ depends on the material properties through the ratio $\beta_{R} / V=\beta_{R} \partial \beta_{R} / \partial \omega$, whose values for $\mathrm{SiC}$, are higher than the corresponding ones obtained for $\mathrm{SiN}$ and $\mathrm{SiO}_{2}$ [Fig. 5(a)] at high frequencies $(\omega / 2 \pi>200 \mathrm{THz})$, their impact on $C$ shows up at high temperature mainly, as established by $\partial f / \partial T$ involved in Eq. (2) and is shown in Figs. 6(a)-6(c) for $T=1000 \mathrm{~K}$. The sharp changes of the spectra for $\mathrm{SiC}$ and $\mathrm{SiO}_{2}$ appear for the frequencies that define the boundaries of the band gaps shown in Fig. 5(b), as expected. For thicknesses $d<500 \mathrm{~nm}$, the maximum contribution to the SPHP heat capacity arises from the vicinity of the frequency $\omega_{p} / 2 \pi=0.05367 T$, as discussed just below Eq. (7). This linear relation of $\omega_{p}$ with $T$ drives the increase of the SPHP heat capacity $C$ with temperature, as shown in Fig. 6(c). One can see that $C$ increases with the film thickness, such that its values for $\mathrm{SiC}$ are generally higher than those of $\mathrm{SiN}$ and $\mathrm{SiO}_{2}$. However, as the film thickness decreases through values $d \leq 100 \mathrm{~nm}$, the SPHP heat capacity becomes independent of $d$ and takes its smallest value $C=C_{0}$, as established by Eqs. (5) and (6a). This fundamental contribution is independent of the material properties and proportional to the square of temperature, as predicted by Eq. (9) and is shown in Fig. 6(c) for $\mathrm{SiO} 2, \mathrm{SiC}$, and $\mathrm{SiN}$. In terms of absolute values, the SPHP heat capacity $C / d \approx 10^{-11} \mathrm{Jcm}^{-3} \mathrm{~K}^{-1}$ (for $\mathrm{SiC}$ at $T=800$ and $d=1 \mu \mathrm{m}$ ) is, however, 11 orders of magnitude smaller than the corresponding typical values of their phonon counterparts (approximately $1 \mathrm{Jcm}^{-3} \mathrm{~K}^{-1}$ ) $[27,28]$ and therefore its values are not expected to be observable in an experiment. The relatively small values of the SPHP heat capacity are generated by the speed of SPHPs, which is close to that of light in vacuum and hence much higher than that of phonons, as established by Eqs. (6a) and (6b). In practice, the contribution of SPHPs to the heat capacity of a polar nanofilm can thus be neglected. For solid materials, such as the ones considered in this work, $(\mathrm{SiO} 2, \mathrm{SiC}$, and $\mathrm{SiN})$, the volumetric heat capacity is usually proportional to the thermal expansion coefficient [36], as established by the experimentally validated Gruneisen relation $[37,38]$. Therefore, the propagation of SPHPs along a thin film is not expected to affect its expansion coefficient, which favors their utilization as heat dissipators.

Finally, note that the considered heat capacity is derived from the variation of the internal energy only (without considering any work [Eq. (2)]) and therefore its values $C=C_{V}$ (SPHP) correspond to the SPHP heat capacity at constant volume. The SPHP heat capacity at constant 
pressure $C_{P}(\mathrm{SPHP})$, on the other hand, can be deduced from Mayer's relation, which establishes that the difference between the total heat capacities at constant pressure $\left(C_{P}\right)$ and volume $\left(C_{V}\right)$ is proportional to the isothermal compressibility $\beta_{T}\left(C_{P}-C_{V} \propto \beta_{T}\right)$ [27,39]. Given that solids are nearly incompressible $\left(\beta_{T} \rightarrow 0\right)$, this relation establishes that $C_{P} \approx C_{V}$, for materials as the ones considered in this work $\left(\mathrm{SiO}_{2}, \mathrm{SiC}\right.$, and $\left.\mathrm{SiN}\right)$. Taking into account that the phonon contributions to the heat capacity at constant volume and pressure of these dielectric materials are nearly identical $\left[C_{P}\right.$ (phonon) $\approx C_{V}$ (phonon)] [27,39], this latter result indicates that their corresponding polariton counterparts are also expected to be nearly equal $\left[C_{P}(\mathrm{SPHP}) \approx\right.$ $\left.C_{V}(\mathrm{SPHP})\right]$.

\section{CONCLUSIONS}

The surface phonon-polariton heat capacity of polar nanofilms is quantified and analyzed as a function of their thickness and temperature. Based on the two-dimensional density of states of surface phonon polaritons, it is shown that their contribution to the heat capacity of nanofilms $(d \leq 100 \mathrm{~nm})$ at sufficiently low temperature $(T<300 \mathrm{~K})$ becomes independent of the material properties and is given by $C=1.15 \varepsilon_{0} k_{B}\left(k_{B} T / \hbar c\right)^{2}$, where $\varepsilon_{0}$ and $c$ are the respective relative permittivity of the surrounding medium and light speed in vaccum, while $k_{B}$ and $2 \pi \hbar$ are the Boltzmann and Planck constants, respectively. Furthermore, it is found that the polariton heat capacity of $\mathrm{SiO}_{2}, \mathrm{SiC}$, and $\mathrm{SiN}$ is several orders of magnitude smaller than its corresponding phonon counterpart, as a result of the relatively high speed of surface phonon polaritons compared to that of phonons.

\section{ACKNOWLEDGMENTS}

This work is supported by the CREST JST Grant No. JPMJCR19Q3 and the project ANR-19-CE09-0005 "EPolariton."

[1] D.-Z. A. Chen, A. Narayanaswamy, and G. Chen, Surface phonon-polariton mediated thermal conductivity enhancement of amorphous thin films, Phys. Rev. B 72, 155435 (2005).

[2] J. Ordonez-Miranda, L. Tranchant, T. Tokunaga, B. Kim, B. Palpant, Y. Chalopin, T. Antoni, and S. Volz, Anomalous thermal conductivity by surface phonon-polaritons of polar nano thin films due to their asymmetric surrounding media, J. Appl. Phys. 113, 084311 (2013).

[3] J. Ordonez-Miranda, L. Tranchant, B. Kim, Y. Chalopin, T. Antoni, and S. Volz, Quantized Thermal Conductance of Nanowires at Room Temperature due to Zenneck SurfacePhonon Polaritons, Phys. Rev. Lett. 112, 055901 (2014).
[4] D.-Z. A. Chen and G. Chen, Heat flow in thin films via surface phonon-polaritons, Front. Heat Mass Transf. 1, 023005 (2010).

[5] Y. Wu, J. Ordonez-Miranda, S. Gluchko, R. Anufriev, D. D. S. Meneses, L. Del Campo, S. Volz, and M. Nomura, Enhanced thermal conduction by surface phononpolaritons, Sci. Adv. 6, eabb4461 (2020).

[6] S. Hamyeh, R. Tauk, P. M. Adam, and M. Kazan, MidIR photothermal measurement of substantial heat transport by surface waves of polar amorphous films supported on silicon, J. Appl. Phys. 128, 095105 (2020).

[7] A. M. Dubrovkin, B. Qiang, H. N. S. Krishnamoorthy, N. I. Zheludev, and Q. J. Wang, Ultra-confined surface phonon polaritons in molecular layers of van der Waals dielectrics, Nat. Commun. 9, 1762 (2018).

[8] A. M. Dubrovkin, B. Qiang, T. Salim, D. Nam, N. I. Zheludev, and Q. J. Wang, Resonant nanostructures for highly confined and ultra-sensitive surface phonon-polaritons, Nat. Commun. 11, 1863 (2020).

[9] D. Li, N. M. Lawandy, and R. Zia, Surface phononpolariton enhanced optical forces in silicon carbide nanostructures, Opt. Express 21, 20900 (2013).

[10] S. A. Holmstrom, T. H. Stievater, M. W. Pruessner, D. Park, W. S. Rabinovich, J. B. Khurgin, C. J. K. Richardson, S. Kanakaraju, L. C. Calhoun, and R. Ghodssi, Guided-mode phonon-polaritons in suspended waveguides, Phys. Rev. B 86, 165120 (2012).

[11] J. D. Caldwell, O. J. Glembocki, Y. Francescato, N. Sharac, V. Giannini, F. J. Bezares, J. P. Long, J. C. Owrutsky, I. Vurgaftman, J. G. Tischler, V. D. Wheeler, N. D. Bassim, L. M. Shirey, R. Kasica, and S. A. Maier, Low-loss, extreme subdiffraction photon confinement via silicon carbide localized surface phonon polariton resonators, Nano Lett. 13, 3690 (2013).

[12] S. Shin and R. Chen, Plasmonically Enhanced Thermal Radiation by Means of Surface Phonon Polaritons, Phys. Rev. Appl. 14, 064013 (2020).

[13] J. Ciers, J. G. Roch, J.-F. Carlin, G. Jacopin, R. Butté, and N. Grandjean, Propagating Polaritons in iii-Nitride Slab Waveguides, Phys. Rev. Appl. 7, 034019 (2017).

[14] D. Z. A. Chen and G. Chen, Measurement of silicon dioxide surface phonon-polariton propagation length by attenuated total reflection, Appl. Phys. Lett. 91, 121906 (2007).

[15] S. Gluchko, B. Palpant, S. Volz, R. Braive, and T. Antoni, Thermal excitation of broadband and long-range surface waves on $\mathrm{SiO}_{2}$ submicron films, Appl. Phys. Lett. 110, 263108 (2017).

[16] F. Yang, J. R. Sambles, and G. W. Bradberry, Long-range surface modes supported by thin films, Phys. Rev. B 44, 5855 (1991).

[17] D. Ma and L. Zhang, Enhancement of interface thermal conductance between $\mathrm{Cr}-\mathrm{Ni}$ alloy and dielectric via $\mathrm{Cu}$ nano-interlayer, J. Phys.: Condens. Mater. 32, 425001 (2020).

[18] D. Ma, A. Arora, S. Deng, G. Xie, J. Shiomi, and N. Yang, Quantifying phonon particle and wave transport in silicon nanophononic metamaterial with cross junction, Mater. Today Phys. 8, 56 (2019).

[19] J. Ordonez-Miranda, L. Tranchant, B. Kim, Y. Chalopin, T. Antoni, and S. Volz, Effects of anisotropy and size of 
polar nano thin films on their thermal conductivity due to surface phonon-polaritons, Appl. Phys. Express 7, 035201 (2014).

[20] J. Ordonez-Miranda, L. Tranchant, T. Antoni, Y. Chalopin, and S. Volz, Thermal conductivity of nano-layered systems due to surface phonon-polaritons, J. Appl. Phys. 115, 054311 (2014).

[21] M. Lim, J. Ordonez-Miranda, S. S. Lee, B. J. Lee, and S. Volz, Thermal-Conductivity Enhancement by Surface Electromagnetic Waves Propagating along Multilayered Structures with Asymmetric Surrounding Media, Phys. Rev. Appl. 12, 034044 (2019).

[22] S. Tachikawa, J. Ordonez-Miranda, Y. Wu, L. Jalabert, R. Anufriev, S. Volz, and M. Nomura, High surface phonon-polariton in-plane thermal conductance along coupled films, Nanomaterials 10, 1383 (2020).

[23] J. Ordonez-Miranda, L. Tranchant, S. Gluchko, and S. Volz, Energy transport of surface phonon polaritons propagating along a chain of spheroidal nanoparticles, Phys. Rev. B 92, 115409 (2015).

[24] E. J. Tervo, O. S. Adewuyi, J. S. Hammonds, and B. A. Cola, High thermal conductivity in polaritonic sio2 nanoparticle beds, Mater. Horiz. 3, 434 (2016).

[25] J. Ordonez-Miranda, L. Tranchant, K. Joulain, Y. Ezzahri, J. Drevillon, and S. Volz, Thermal energy transport in a surface phonon-polariton crystal, Phys. Rev. B 93, 035428 (2016).

[26] L. Tranchant, S. Hamamura, J. Ordonez-Miranda, T. Yabuki, A. Vega-Flick, F. Cervantes-Alvarez, J. J. Alvarado-Gil, S. Volz, and K. Miyazaki, Two-dimensional phonon polariton heat transport, Nano Lett. 19, 6924 (2019).

[27] N. W. Ashcroft and N. D. Mermin, Solid State Physics (Harcout College Publishers, Forth worth, Philadelphia, 1976).

[28] G. Chen, Nanoscale Energy Transport and Conversion: A Parallel Treatment of Electrons, Molecules, Phonons, and Photons (Oxford University Press, New York, 2005).

[29] V. M. Agranovich, Surface Polaritons (Elsevier, Amsterdam, 2012).
[30] J. D. Caldwell, L. Lindsay, V. Giannini, I. Vurgaftman, T. L. Reinecke, S. A. Maier, and O. J. Glembocki, Low-loss, infrared and terahertz nanophotonics using surface phonon polaritons, Nanophotonics 4, 44 (2015).

[31] A. M. Hofmeister, J. Dong, and J. M. Branlund, Thermal diffusivity of electrical insulators at high temperatures: Evidence for diffusion of bulk phonon-polaritons at infrared frequencies augmenting phonon heat conduction, J. Appl. Phys. 115, 163517 (2014).

[32] P. Li, X. Yang, T. W. W. Mab, J. Hanss, M. Lewin, A.-K. U. Michel, M. Wuttig, and T. Taubner, Reversible optical switching of highly confined phonon-polaritons with an ultrathin phase-change material, Nat. Mat. 15, 870 (2016).

[33] S. Dai, Q. Ma, Y. Yang, J. Rosenfeld, M. D. Goldflam, A. McLeod, Z. Sun, T. I. Andersen, Z. Fei, M. Liu, Y. Shao, K. Watanabe, T. Taniguchi, M. Thiemens, F. Keilmann, P. Jarillo-Herrero, M. M. Fogler, and D. N. Basov, Efficiency of launching highly confined polaritons by infrared light incident on a hyperbolic material, Nano Lett. 17, 5285 (2017).

[34] I. Dorofeyev, On the equilibrium spectrum of surface polaritons thermally excited by solids, Phys. Lett. A 375, 2885 (2011).

[35] Karl Joulain, Younès Ezzahri, Jérémie Drevillon, Benoît Rousseau, and Domingos De Sousa Meneses, Radiative thermal rectification between $\mathrm{SiC}$ and $\mathrm{SiO}_{2}$, Opt. Express 23, A1388 (2015).

[36] J. Garai, Correlation between thermal expansion and heat capacity, Calphad 30, 354 (2006).

[37] D. McLachlan and W. R. Foster, The relationship between coefficients of expansion and heat capacities of simple metals, J. Solid State Chem. 20, 257 (1977).

[38] V. A. Drebushchak and A. I. Turkin, Relationship between heat capacity and thermal expansion derived from the lennard-jones potential, J. Therm. Anal. Cal. 65, 745 (2001).

[39] C. Kittel and H. Kroemer, Thermal Physics (W. H. Freeman, New York, 1980). 